\title{
Perpendicular Fluid Flow Measurement with a Spatial Array of Ultrasonic Transducers
}

\author{
James S. Hall \\ Electrical and Computer Engineering \\ Georgia Institute of Technology \\ Atlanta, GA, USA \\ jshall@gatech.edu
}

\begin{abstract}
The challenge of remotely measuring fluid flow through a plane of interest for a wide range of velocities is addressed using an array of ultrasonic transducers. Unlike traditional transit-time ultrasonic flow measurements, the proposed method uses the angle-of-arrival of a reflected ultrasonic wave to measure flow perpendicular to the path of propagation. The use of angle-of-arrival information avoids the inherent requirement of transit-time techniques to have a nonnegligible directional component of the propagation path in the flow direction. A successful proof-of-concept was developed to experimentally validate the proposed technique.
\end{abstract}

Keywords-velocity measurement, beamforming, phased array, ultrasonics

\section{INTRODUCTION}

This paper addresses the challenge of remote fluid flow measurement through a plane of interest for a wide range of velocities. Remote measurement, in this context, refers to an ability to physically measure (not infer) the fluid flow through a plane without physically placing a sensor in the center. In other words, any sensors must be located on the periphery of the plane, ideally co-located to simplify installation. The velocities of interest span from that typically considered very slow $(<1 \mathrm{~m} / \mathrm{s})$ to those typical of hurricanes $(>70 \mathrm{~m} / \mathrm{s})$.

Many techniques are commercially available for fluid flow measurement. Mechanical methods, such as vane or cup anemometers, are limited to measuring flow local to the sensor and can only measure velocities down to the point at which the fluid force functioning on the sensor cannot overcome the sensor's frictional forces. Thermal techniques, such as hot wire anemometers [1] and thermal mass flow meters [2], are also limited to measuring the flow locally. Laser and ultrasonic Doppler techniques [3-5] can measure flow remotely, but require scatterers to be present in the fluid and can be prohibitively expensive. Ultrasonic transit-time techniques $[6,7]$ represent an inexpensive means of measuring fluid flow remotely; however, these techniques estimate the flow rate by measuring a change in propagation velocity, which requires that the propagation path have some nonnegligible directional component in the flow direction. As

This work has been supported by NASA's Graduate Student Research Program (GSRP) Grant No. NNX08AY93H. such, ultrasonic transit-time techniques cannot be used directly to measure fluid-flow through a plane of interest.

The proposed method uses a spatial array of transducers to estimate the angle-of-arrival of a reflected ultrasonic wave and thus remotely measure average flow perpendicular to the path of propagation. Because the angle-of-arrival is used to determine the flow velocity, the range of velocities that can be measured is limited only by the system's calibration and stability.

The paper is organized as follows: Section II briefly discusses the proposed method for flow measurement. Section III describes a proof-of-concept experiment conducted to validate the use of a spatial array for flow measurement, including a description of the experimental setup, challenges, and results. Finally, Section IV concludes the paper with a brief summary and recommendations for future work.

\section{VELOCITY MEASUREMENT}

Velocity measurement with an ultrasonic array takes advantage of the array's ability to estimate an incident wave's angle-of-arrival. As shown in Fig. 1, the basic setup involves an ultrasonic array, which performs both signal transmission and reception, and a reflector. Velocity measurement starts by transmitting a pulse into the flow medium. The ultrasonic signal will propagate across the flow, reach the reflector opposite the flow, and then return to the array. Upon receiving the reflected signal, the ultrasonic array can measure the angle-of-arrival.

Note that this approach to flow measurement can be generalized to accommodate angled reflectors, which have a non-zero angle-of-arrival for the no-flow case. However, in the interest of space, the derivation of the more general case is foregone in favor of a perpendicular reflector, which conveys the fundamental concept.

Figure 1(a) provides graphical representation of the reflected wave as it impinges on the transducer array when no flow is present. In contrast, Fig. 1(b) depicts the case when a flow is present. Figure 1(c) shows a velocity vector diagram that illustrates how the perpendicular flow can be estimated based on the angle-of-arrival measurement and assumed 
(a) Reflector
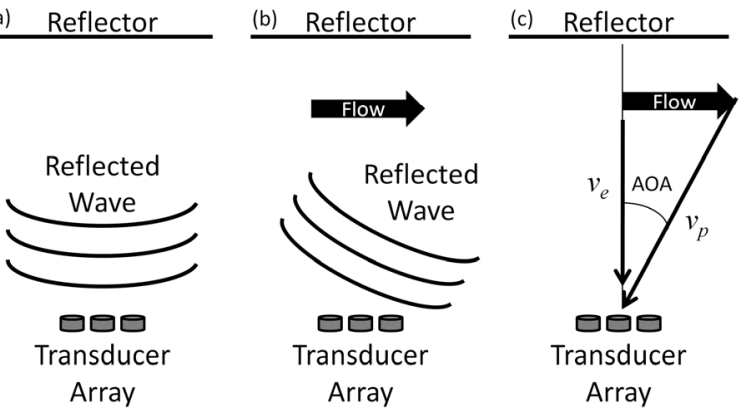

Figure 1. Velocity measurement diagrams. (a) Reflected wave in no-flow scenario. (b) Reflected wave with flow present. (c) Velocity vector diagram.

propagation velocity. Since the longitudinal wavespeed of the ultrasonic signal is consistent throughout the medium, the Law of Sines can be used to determine the flow rate under the assumption that the flow is uniform over the propagation distance:

$$
f_{u l t}=v_{p} \sin ^{-1}(A O A),
$$

where $f_{u l t}$ is the flow velocity measured over the propagation distance, $v_{p}$ is the longitudinal wavespeed, and $A O A$ is the angle-of-arrival measured by the transducer array.

It is important to point out here that the flow measurement, $f_{u l t}$, is not a traditional average of the flow profile, $f(x)$, which describes average perpendicular flow as a function of distance. Let the effective velocity of the wavefront be defined as:

$$
v_{e}=\sqrt{v_{p}^{2}-f_{u l t}^{2}} .
$$

Assuming that the propagation path is perpendicular to both the transducer array and flow direction, as in Fig. 1, the timeof-arrival, $t_{o a}$, can be expressed in terms of $v_{e}$ :

$$
t_{o a}=\frac{2 d}{v_{e}}=2 \int_{0}^{d} \frac{d x}{v_{e}(x)},
$$

where $d$ represents the distance from transducer array to reflector, and $v_{e}(x)$ is the effective velocity as a function of distance from the transducer array. Substituting Eq. (2) into Eq. (3) and solving for the flow measurement, $f_{u l t}$, results in:

$$
f_{u l t}=\sqrt{v_{p}^{2}-\frac{d^{2}}{\left(\int_{0}^{d} \frac{d x}{\sqrt{v_{p}^{2}-f^{2}(x)}}\right)^{2}}} .
$$

Eq. (4) reflects the complicated relationship between the flow velocity measured with an ultrasonic array, $f_{u l t}$, as a function of the flow velocity profile, $f(x)$.

\section{EXPERIMENTAL PROOF-OF-CONCEPT}

\section{A. Experimental Setup}

Figure 2 illustrates the experimental setup. A $14 \mathrm{~cm}$ diameter, $2 \mathrm{~m}$ long hard plastic tube was used to condition the flow, mount the transducers, and reflect the ultrasonic waves. Air flow was induced with two different commercial fans located approximately $1 \mathrm{~m}$ from the nearest tube end. Flow rate was adjusted by restricting the tube opening closest to the

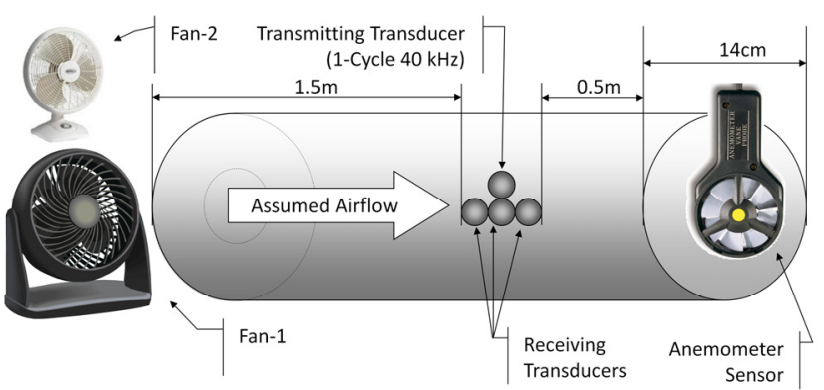

Figure 2. Experimental Setup. Two commercial fans generated flow. Velocities were adjusted by restricting tube opening. A vane anemometer validated array flow measurements.

flow source. At the other end of the tube, a vane-anemometer was used to independently measure the flow.

The transducers were arranged with a single transmitter, located adjacent to the center of the 3 -element receiving array. A single-cycle $40 \mathrm{kHz}$ pulse was used for excitation.

The experiment was conducted in four stages. First, numerical simulations were performed to verify the feasibility of the setup. Then, during the second stage, calibration data were collected with both ends of the tube closed to prevent flow. The final two stages involved flow measurements specific to each commercial fan. For each of the flow measurement stages, pre-calibration and post-calibration data were obtained before and after flow measurements were taken. Each velocity measurement was performed by adjusting the opening size, allowing the flow to stabilize, and then taking measurements with both the ultrasonic array and reference anemometer. The opening sizes were applied in a randomized sequence that differed between the two stages.

\section{B. Flow Measurement Comparison}

The velocity profile inside the tube must be accounted for in order to compare flow measurements from an ultrasonic array and measurements from an anemometer. For flow corresponding to a Reynolds number greater than 4000 , which is the case for the experimental setup, a turbulent flow is expected. Under turbulent flow conditions, the time-averaged flow velocity is approximated using a power-law profile [8]:

$$
f_{\text {turb }}(r)=v_{\max }\left(1-\frac{r}{R}\right)^{\frac{1}{N}}
$$

where $N$ is typically assumed to be $7, v_{\max }$ is the maximum velocity of the flow profile, and $R$ is the tube radius.

The anemometer measurement, $f_{a n}$, represents the average velocity over the surface area of the anemometer sensor:

$$
f_{a n}=\frac{2}{R_{a n}^{2}} \int_{0}^{R_{a n}} r f(r) d r
$$

where $R_{a n}$ is the radius of the anemometer and $f(r)$ is the assumed velocity profile, as in Eq. (5). This is an entirely different function than that of the ultrasonic array, which is described in Eq. (4). Therefore, the anemometer and ultrasonic array are expected to measure different velocities 
because they are measuring different portions of the same flow profile.

An assumed velocity profile provides a means to compare the two measurements. For the proof of concept described here, the anemometer measurements taken over a subset of the velocity profile are converted into "equivalent" ultrasonic measurements, taking into account the entire velocity profile. First, an estimate of the maximum velocity, $v_{\max }$, is obtained from the anemometer measurement by assuming a turbulent flow, substituting Eq. (5) into Eq. (6), and solving for $v_{\max }$ :

$$
v_{\max }=\frac{f_{a n} R_{a n}^{2}}{2 R^{2} N\left(\frac{1-\left(1-\frac{R_{a n}}{R}\right)^{\frac{N+1}{N}}}{N+1}+\frac{\left(1-\frac{R_{a n}}{R}\right)^{\frac{2 N+1}{N}}-1}{2 N+1}\right) .}
$$

Rather than attempt to repeat the analogous substitution and solve for $v_{\max }$ using Eq. (4), which does not easily lend itself to a closed form solution, $v_{\max }$ obtained from Eq. (7) is substituted into Eq. (5) to obtain an estimate of the velocity profile. The velocity profile estimate can then be substituted into Eq. (4) to obtain an estimate of the "equivalent" ultrasonic measurement. From here, the volumetric flow rate is approximated by a simple multiplication:

$$
V=\pi R^{2} f_{\text {ult }} \text {. }
$$

\section{Experimental Challenges}

1) Near-Field Effects: Many spatial-array applications function under an assumption that the incident wave is planar [9] (a.k.a. the far-field assumption). This assumption allows array steering to be performed with simple phase adjustments to the received signals. In this proof-of-concept, however, the propagation distance is very short, which means that the incoming wavefront will have a spherical curvature. Therefore, phase shifts were calculated as both a function of the propagation path distance, $d$, and potential flow rate, $f$.

2) Calibration and System Stability: Numerical simulations performed during the first experimental stage indicated that the relative transducer locations and propagation velocity are critical to flow measurement accuracy. Therefore, a data-driven calibration method was developed to characterize the experimental environment. Calibration signals were recorded during the second stage of the experiment without flow through the tube. The calibration algorithm assumed a model of the experimental setup that included the following parameters: transducer locations, propagation speed, and cylinder diameter. A non-linear search was then performed to find the parameters that allowed the model to most closely agree with the calibration data. These parameters were then used in the angle-of-arrival calculations for flow measurement.

System phase stability issues were observed during the experiment as well. When the tube opening closest to the fan was completely closed, the flow measurement is expected to be zero. However, during the pre- and post-calibration of the flow measurement stages, non-zero flow was measured with the ultrasonic array. These non-zero flow measurements indicate disagreement with the calibration data and are attributed to system phase instability. To compensate, a phase shift is applied to the received signals so that the pre- and postcalibration flow measurements are zero. Since the pre-and post-calibration measurements were found to be different, it was assumed that the phase changed linearly throughout the flow measurements.

3) Multipath and Ringing Effects: The use of a cylindrical environment introduces multipath effects where the received signal, in addition to containing the signal reflected from the opposite side of the tube, also contains the signal that was reflected from the tube walls twice, three times, etc. This problem is easily mitigated by using a short-duration excitation signal and focusing on signals that arrive without interference. However, since the transducers that were used for this experiment exhibited a substantial amount of ringing at the chosen operating frequency, the signal duration could not be sufficiently reduced to avoid interference. As a result, the direct-arrival and multipath reflections were found to be overlapping in the experimental signals. Figures 3(a) and 3(b) demonstrate the simulated and actual time-domain signals, respectively.

The problem of overlapping reflections was addressed by analyzing the phase relationship between received signals. As shown in Fig. 3(c), there is a period of time in which the phase relationship between received signals is stable. Even though this time period likely corresponds to overlapping reflections and not the direct arrival, these stable phase periods can still be used to estimate the angle-of-arrival. This can be seen in Fig. 1 and Eq. (1), where the flow velocity measurement is independent of the distance traveled and the number of reflections.

The use of signals that do not correspond to the anticipated direct arrival is problematic in two respects. First, the wavefront will not have the anticipated curvature, which will undoubtedly introduce some error into the angle-of-arrival
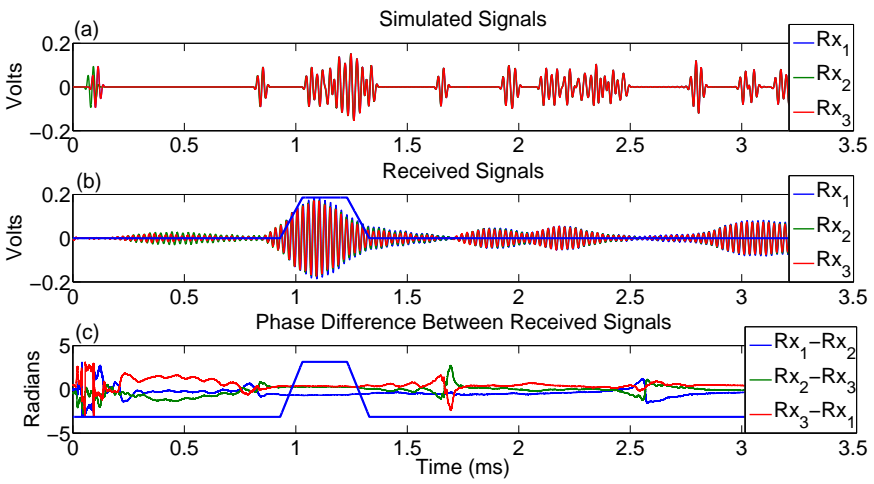

Figure 3. Multipath and ringing effects. Excessive ringing from both the transmitting and receiving transducer obscure the expected direct arrival. (a) Simulated multipath signals. (b) Actual multipath signals. (c) Phase relationship between receivers. The window indicated in (b) and (c) indicates window used to isolate stable phase relationships. 
measurement. The second problem is that since the path corresponds to overlapping reflections, the actual path traveled is unknown. This affects the ability to properly validate the measurements with an assumed velocity profile.

4) Velocity Profile: For the experimental setup, based on the Reynolds numbers calculated for the pipe and measured flow rates, turbulent flow is expected. However, turbulent flow requires an entry length of $4.4 D \mathrm{Re}^{1 / 6}$ to become fully developed [8], where $\mathrm{D}$ is the tube diameter and $\mathrm{Re}$ is the Reynolds number. Unfortunately, the experimental entry length is only 10 diameters and the Reynolds numbers exceed 4000 for all but the slowest velocities measured. Therefore, the flow is not fully developed and the assumed flow velocity profile may be inaccurate.

\section{Experimental Results}

Volumetric flow measurement estimates were obtained from the anemometer and ultrasonic array measurements as described in the previous section. Experimental flow measurements are shown in Fig. 4. Anemometer measurements are shown with error bars set according to manufacturer established bounds. Three key observations can be made from Fig. 4: (1) The ultrasonic array measurements are in relative agreement with each other, meaning that as the flow is decreased/increased, the ultrasonic array measurement is likewise decreased/increased. (2) The ultrasonic array measurements are in reasonable agreement with the anemometer measurements. (3) The ultrasonic array appears to continue to measure flow at speeds below the range of the anemometer.

Discrepancies between the ultrasonic array and anemometer measurements are primarily attributed to four factors: (1) Since the turbulent flow is not fully-developed, the use of a power-law velocity profile is inaccurate. The use of a valid velocity profile is critical to measurement comparison. (2) The use of overlapping echoes will negatively affect the ability to determine the angle-of-arrival because the spherical wavefront for each echo will have a slightly different curvature. (3) Anemometer measurements are adjusted based on the assumption that the ultrasonic propagation path is directly through the center of the tube. However, the echoes selected for this experiment are representative of waves that reflected more than once before returning to the sensor array. As a result, the volumetric flow estimates for the anemometer reflected in Fig. 4 reflect a higher value than would be expected if the actual propagation path were accounted for. (4) Velocity measurements taken with a linear array implicitly assume that the flow being measured lies in the plane of the array. In turbulent conditions, however, especially when the transmitted and reflected wavefronts do not propagate in opposite directions along the same path, some flow components will not be parallel to the array. This will introduce additional error in the angle-of-arrival estimate.
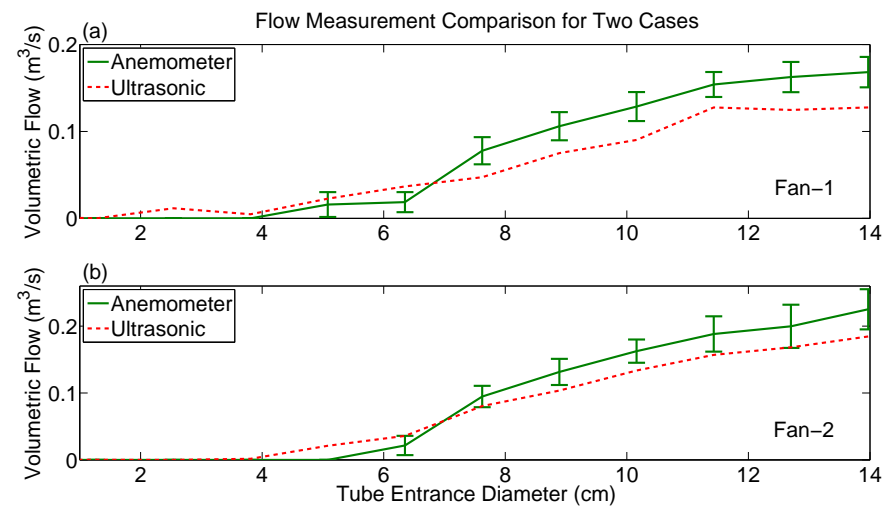

Figure 4. Volumetric flow measurements. Anemometer measurements were adjusted as described in Section III-B. Error bars are based on manufacturer's stated accuracy. Volumetric flow assumes a turbulent velocity profile. (a) Flow generated with Fan-1. (b) Flow generated with Fan-2.

\section{SUMMARY AND CONCLUSION}

The use of an ultrasonic spatial array was proposed for measuring perpendicular fluid flow. This technique has the potential for fast, accurate measurements along the entire propagation path for a wide range of velocities. System accuracy is limited only by calibration accuracy and system stability. A successful proof-of-concept was implemented in which experimental flow measurement results agreed reasonably well with reference measurements taken with a vane anemometer.

Future work should be directed towards experimental verification over a wider range of velocities with a system that avoids some or all of the experimental challenges discussed in this paper.

\section{ACKNOWLEDGMENT}

The author would like to thank Dr. Robert Youngquist and Dr. Janine Captain at NASA's Kennedy Space Center for their guidance and generous support with experimental validation.

\section{REFERENCES}

[1] H. Bruun, Hot-Wire Anemometry: Oxford University, 1995.

[2] S. A. Tison, "A critical evaluation of thermal mass flow meters," J. Vac. Sci. Technol. A, vol. 14, no. 4, pp. 2582-2591, 1996.

[3] P. D. Iten, Laser Doppler Anemometer, United States 3,966,324, 1976.

[4] T. L. Szabo, Diagnostic Ultrasound Imaging: Inside Out, Burlington, MA: Elsevier Academic Press, 2004.

[5] T. R. Lawrence, D. J. Wilson, C. E. Craven et al., "A laser velocimeter for remote wind sensing," Review of Sci. Inst., vol. 43, no. 3, pp. 512$518,1972$.

[6] P. Hiismaki, Acoustic Flow Measurement Method and Measurement Apparatus Implementing the Method, United States 5,770,806, 1998.

[7] B. Pincet, P. Journe, and G. Brugnot, Ultrasonic Anemometer, United States 4,890,488, 1990.

[8] B. R. Munson, D. F. Young, and T. H. Okiishi, Fundamentals of Fluid Mechanics: John Wiley \& Sons, 2002.

[9] D. H. Johnson, and D. E. Dudgeon, Array Signal Processing: Concepts and Techniques: Prentice Hall, 1993. 